\title{
Molecular scale chemical imaging of the orientation of an on-surface coordination complex by tip-enhanced Raman spectroscopy
}

\author{
Zhen-Feng Cai, ${ }^{1 \dagger}$ Li-Qing Zheng, ${ }^{1 \dagger}$ Yao Zhang, ${ }^{2 *}$ and Renato Zenobi ${ }^{1 *}$ \\ ${ }^{1}$ Department of Chemistry and Applied Biosciences, ETH Zurich, Zurich, Switzerland. \\ ${ }^{2}$ Hefei National Laboratory for Physical Sciences at the Microscale and Synergetic Innovation Center of Quantum Infor- \\ mation and Quantum Physics, University of Science and Technology of China, Hefei, Anhui 230026, China.
}

\begin{abstract}
Metal-organic coordination structures at interfaces play an essential role in many biological and chemical systems. Understanding the molecular specificity, orientation and spatial distribution of the coordination complexes at the nanometer scale is of great importance for effective molecular engineering of nanostructures and fabrication of functional devices with controllable properties. However, fundamental properties of such coordination systems are still rarely studied directly. In this work, we present a spectroscopic approach on the basis of tip-enhanced Raman spectroscopy (TERS) to investigate a cobalt(II) tetraphenyl-porphyrine (CoTPP) coordination species on the scale of a single molecule under ambient conditions. Coordination species anchored on gold surfaces modified with pyridine thiol self-assembled monolayers can be spectroscopically distinguished and mapped with $c a$. $2 \mathrm{~nm}$ resolution. In addition, in combination with density functional theory simulations, the adsorption configuration and molecular orientation of the coordination complexes are also revealed using TERS imaging.
\end{abstract}

\section{INTRODUCTION}

Bottom-up fabrication of functional metal-organic structures on surfaces provides coordination systems suitable for developing devices with controllable properties via different combinations and spatial arrangements of building blocks. ${ }^{1}$ Metalloporphyrins (MPs) and metallophthalocyanines (MPcs) have attracted great interest because of their remarkable catalytic properties and have been widely applied in many biological and chemical systems. ${ }^{2}$ Using self-assembled monolayers (SAMs) as anchors to immobilize MPs/MPcs on surfaces through coordination interactions is one of the important approaches to fabricate functional films for various applications like click reactions, oxygen reduction reaction, photovoltaic devices, etc. ${ }^{3}$ A SAM coordinating with MPs/MPcs promotes the catalytic activities of a pure MP/MPc molecular catalysts via electron transfer mechanism. ${ }^{4}$ However, fundamental details related to the molecular specificity, configuration and orientation are still poorly explored on the nanoscale, though it is of great importance for the understanding of structurereactivity relationships.

Scanning tunneling microscopy (STM) has been demonstrated to be a powerful tool for the study of on-surface self-assemblies at the molecular level. ${ }^{5}$ However, for such metal-organic species on SAMs, STM is not able to reveal chemical information about the organic components and molecular orientations. ${ }^{6}$ In some cases the template sequence has to be reverted to create a relatively flat bottom layer for molecular imaging. ${ }^{7}$ For instance, on-surface coordination of MPs with $\mathrm{N}$-heterocyclic carbenes ${ }^{7 \mathrm{a}}$, imidazole ${ }^{7 \mathrm{~b}}$, pyridine ${ }^{7 \mathrm{c}}$, and triethylenediamine ${ }^{7 \mathrm{~d}, 8}$ has been studied via contrast changes in STM imaging; high-contrast species are assigned to coordination complexes with the help of theoretical simulations. Nevertheless, it is still a challenge to visualize the coordination species on surfaces with chemical specificity and detailed information at the molecular level under ambient conditions.

Tip-enhanced Raman spectroscopy (TERS) has been applied to obtain chemical fingerprint and topographic information of various organic layers by taking advantage of its single molecule sensitivity and ultrahigh spatial resolution. ${ }^{9}$ TERS allows for the unambiguous chemical identification of individual molecules assembled on a surface and provides vibrational fingerprints that are very sensitive to the molecular configuration and orientation. In some model systems, it has been shown that TERS can spatially resolve molecular species on a surface with $c a .3 \mathrm{~nm}$ resolution at ambient conditions ${ }^{10}$, but it is still challenging to achieve molecular scale TER imaging in ambient environments. In recent years, STM-TERS has also been applied to achieve chemical identification of MPs/MPcs and study chemical processes occurring in corresponding SAMs. For example, single-molecule TER imaging of single- and dual-component MPs/MPcs on surfaces has been demonstrated in ultrahigh vacuum and of cryogenic temperatures with sub-nanometer resolution. ${ }^{11}$ Under electrochemical conditions, STMTERS was applied to study the MPc-catalyzed oxygen reduction reaction on single crystal electrodes, through which the identification of reactive intermediates and products formed by the coordination of the metal center with reactive species such as $\mathrm{O}_{2}, \mathrm{HO}_{2}^{-}, \mathrm{OH}^{-}, \mathrm{O}_{\text {was }}$ achieved. ${ }^{12}$ However, to the best of our knowledge, the study of a MPs/MPcs based coordination system on the molecular scale using ambient STM-TERS has not been achieved yet.

Here, we employed ambient STM-TERS (Figure 1a) to investigate the formation and molecular orientation of a 5,10,15,20-tetraphenyl$21 \mathrm{H}, 23 \mathrm{H}$-porphyrine cobalt(II) (CoTPP)-4-mercaptopyridine (4PySH) coordination system immobilized on Au(111) and template-striped 
gold (TS-Au) surfaces (Figure $1 \mathrm{~b}$ and c) with a spatial resolution of $c a .2 \mathrm{~nm}$. TERS maps showed the spatial distribution of CoTPP on a bicomponent thiolate buffer layer with different molecular ratios. Furthermore, combining TERS maps with density functional theory (DFT) simulations, we are able to determine the molecular orientation of CoTPP in the coordination system on the Au(111) substrate. The present work broadens the application of TERS for studying on-surface coordination chemistry and related dynamics at the nanoscale.

\section{RESULTS AND DISCUSSIONS}

Figure 1d shows the confocal Raman spectrum of CoTPP and the TER spectra of 4PySH and CoTPP on different Au substrates. It can be observed that when CoTPP is directly adsorbed on the Au(111) surface, the TER spectrum of CoTPP is different from its powder Raman spectrum. In the TER spectrum, the peak intensity is relatively weak and only few broad peaks can be observed. Such spectral differences are in good agreement with previous work ${ }^{13}$ and can be attributed to the surface selection rules of TERS. ${ }^{11 \mathrm{c}}$ In contrast, when a well-covered 4PySH monolayer (Figure S1) was applied to anchors CoTPP, the peak intensity of CoTPP increases dramatically and most of the bands are in very good agreement with the powder Raman spectrum of CoTPP (outlined by grey dots). This indicates that the on-surface coordination relaxes the selection rules, and makes most of the vibration bands from the porphyrin visible because the buffer layer greatly decrease the substrate confinement by weakening the surface-molecule interactions. In addition, shifts of the vibrational modes at $c a .1000 \mathrm{~cm}^{-1}$ and 1250 $\mathrm{cm}^{-1}$ are observed (labeled by blue dash-dots), due to the coordination effect on the vibration of the porphyrin core and phenyl rings. On the other hand, the overall intensity of the vibrational bands of 4PySH below CoTPP is suppressed. They can only be distinguished as small bands or shoulder peaks, as highlighted by the green dashes, which could be explained by the inhomogeneous distribution of the field enhancement between the Ag tip and the Au substrate. ${ }^{14}$

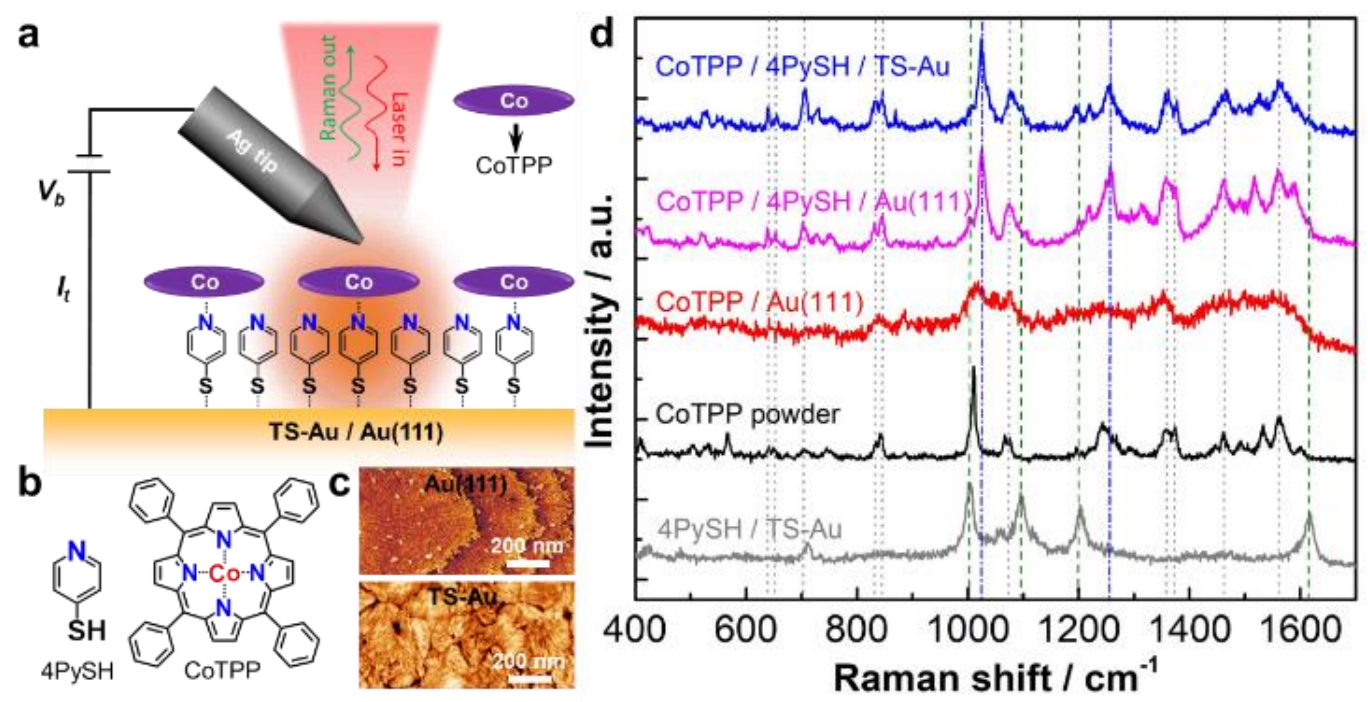

Figure 1. (a) Schematic of STM-TERS probing the CoTPP-4PySH coordination interaction on a Au surface using a Ag tip. (b) Molecular structures of 4PySH and CoTPP. (c) Large-scale STM images of bare Au(111) and bare TS-Au surfaces. (d) TER spectra of CoTPP modified Au(111) (red trace), CoTPP on 4PySH-modified-Au(111) (pink trace) and 4PySH-modified-TS-Au (blue trace). For comparison, a confocal powder Raman spectrum of CoTPP (black trace) and a TER spectrum of a $4 \mathrm{PySH}$ monolayer on TS-Au (grey trace) are also displayed.

In order to verify the coordination interaction between 4PySH and CoTPP, the 4PySH monolayer was replaced by a $2 \mathrm{PySH}$ adlayer on the $\mathrm{Au}$ surface to support CoTPP. As shown in Figure S2, only spectroscopic features from the 2PySH molecules can be detected after applying CoTPP molecules on top of a 2PySH-modified Au surface after rinsing with ethanol, which confirms that CoTPP molecules were mainly stabilized on the 4PySH-modified Au surface via coordination interaction between the $\mathrm{N}$ atom of $4 \mathrm{PySH}$ and the Co atom of CoTPP, rather than by van der Waals interactions.

Taking advantage of the different adsorption behavior of CoTPP on 4PySH and 2PySH adlayers, a mixed buffer layer consisting of 4PySH and 2PySH was applied as a model system to facilitate the spectroscopic visualization of the formation and spatial distribution of on-surface coordination species (Figure 2a). The mixed buffer layer modified TS-Au substrate was prepared by immersing the Au substrate into an ethanolic solution containing 4PySH and $2 \mathrm{PySH}$. Detailed spectroscopic visualization of surface species and the complementary relationship of the spatial distribution of $4 \mathrm{PySH}$ and $2 \mathrm{PySH}$ on Au are shown in Figure S3. After adding CoTPP molecules to the 2PySH-4PySH mixed adlayer, TERS imaging was performed. Figure $2 b, c$ shows a full spectral map, as well as the TER spectra extracted from the positions indicated by black stars on the map. At positions P1 and P2, vibrational modes of CoTPP-4PySH coordination clearly appeared, consistent with the TER spectrum of CoTPP on the 4PySH modified Au substrate. The Raman fingerprints at positions P3 and P4 are totally different 
from those of P1/P2, where mainly the features of 2PySH molecules were observed. For positions P5 and P6, Raman features in good agreement with the experimental TERS results of $4 \mathrm{PySH}$ molecules can be clearly observed. These results show that the spatial distribution of the tri-component system including coordination species can be spectroscopically well resolved at the nanometer scale by STM-TER imaging.
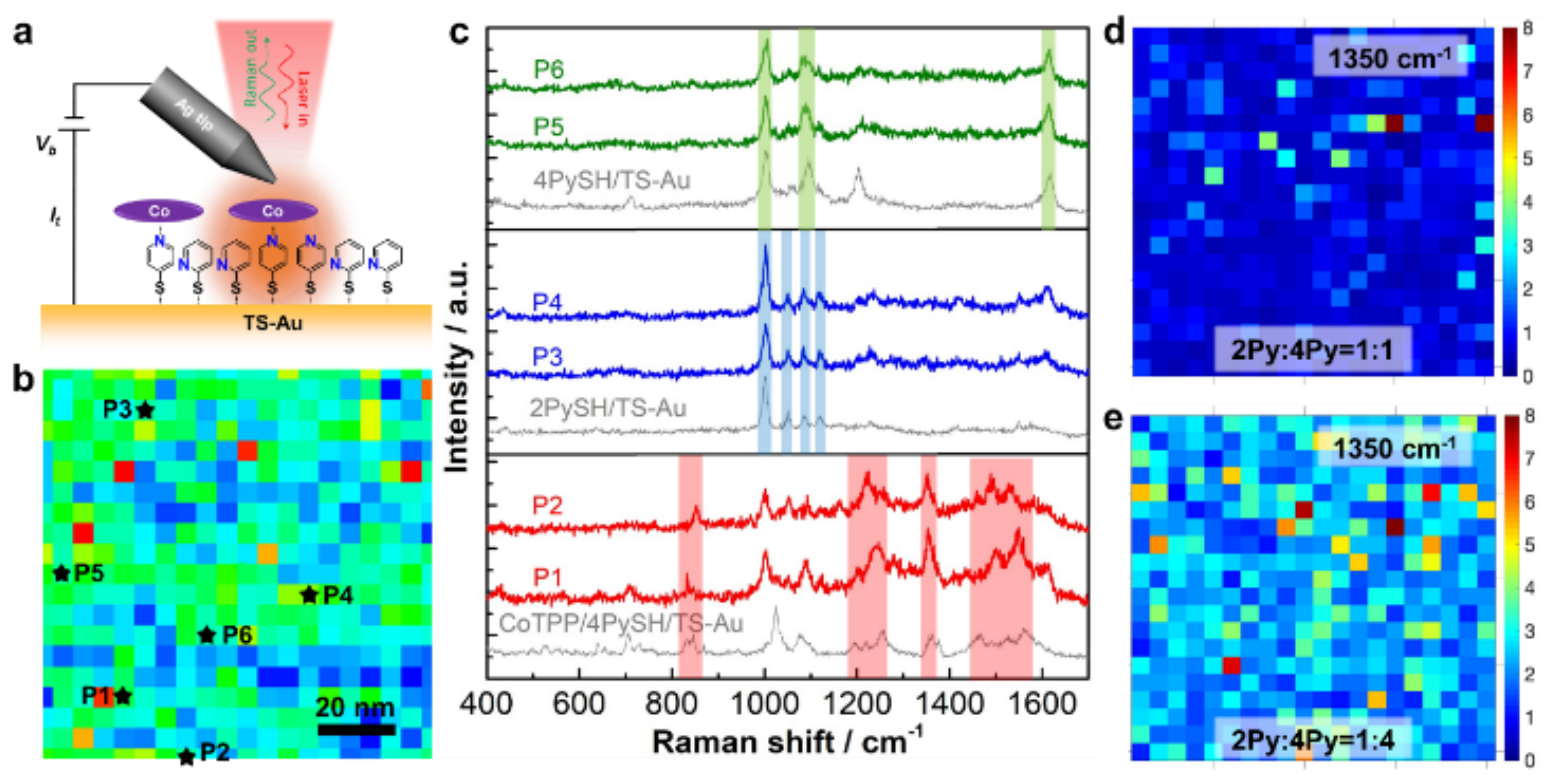

Figure 2. (a) Schematic of STM-TERS probing of CoTPP species on the TS-Au surface modified with a 2PySH and 4PySH mixed adlayer. (b) TERS intensity map of the full spectrum of CoTPP on a (2PySH-4PySH)-modified TS-Au surface. The size of the map is $100 \times 100 \mathrm{~nm}^{2}$ with a pixel size of $5 \mathrm{~nm}$. (c) Corresponding TER spectra at the positions marked in $\mathbf{b}$ and the reference TER spectra (grey traces). (d, e) TERS peak intensity map $\left(1350 \mathrm{~cm}^{-1}\right)$ of CoTPP on (2PySH-4PySH) modified Au surfaces with different 2PySH/4PySH ratios. The molar ratio of 2PySH and 4PySH is 1:1 (d) and 1:4 (e), respectively. The size of the maps are $1000 \times 1000 \mathrm{~nm}^{2}$ with a pixel size of $50 \mathrm{~nm}$.

Since the peak at $1350 \mathrm{~cm}^{-1}$ is one of the characteristic peaks of CoTPP, we choose it to map the spatial distribution of the coordination complex on the mixed buffer layer modified TS-Au surface. Figure $2 \mathrm{~d}$ shows the peak intensity map of the $1350 \mathrm{~cm}^{-1} \mathrm{TERS}$ signal of a sample prepared from a mixed solution with a ratio of $2 \mathrm{PySH}: 4 \mathrm{PySH}=1: 1$. Only few pixels on the map shows the signal at $1350 \mathrm{~cm}^{-1}$ while most of the positons display as low-contrast features, which suggests that relatively few CoTPP-4PySH species are formed on the surface. In order to further confirm the spectroscopically resolved spatial distribution, a mixed 2PySH-4PySH adlayer with a higher ratio of 4PySH was prepared from a mixed solution with a ratio of $2 \mathrm{PySH}: 4 \mathrm{PySH}=1: 4$ and then functionalized with CoTPP molecules. Figure 2e shows that with an increased fraction of 4PySH molecules on the surface, the spectroscopic contrast of the Raman band at $\sim 1350-1370 \mathrm{~cm}^{-1}$ also becomes much higher and can be detected at more positions, which clearly shows the presence of CoTPP-4PySH coordination species on the TS-Au surface compared with that on the buffer adlayer made from $2 \mathrm{PySH}$ and $4 \mathrm{PySH}$ with a molar ratio of 1:1. It is interesting to note that the selective complexation of CoTPP on 4PySH can also act as a probe to visualize the spatial distribution of both thiolate molecules on gold. Data on different molecular ratios of CoTPP on 2PySH-4PySH modified Au surfaces are shown in Figure S4 and the step-by-step increase of CoTPP-4PySH complex can be observed with the increase of on-surface molecular ratio of $4 \mathrm{PySH}$.

Having established that the on-surface coordination species can be distinguished spectroscopically on the surface, we further performed highresolution imaging to explore the spatial resolution of our current system. Because the thermal drift of the system was $0.02-0.03 \mathrm{~nm} / \mathrm{s}$ under laser illumination in ambient conditions (Figure S5), short acquisition time (1 s) was chosen to reduce the effect of drift. Figure 3 shows the TERS map and the corresponding TER spectra extracted from the map at the marked pixels. Surprisingly, when the pixel size was decreased to $2 \mathrm{~nm}$, CoTPP-4PySH coordination complex still can be resolved clearly. As shown in Figure 3b, the appearance of the CoTPP-4PySH complex is indicated by the appearance of the distinctive bands at $\sim 835 \mathrm{~cm}^{-1}, \sim 1240-1260 \mathrm{~cm}^{-1}$, and $\sim 1350-1370 \mathrm{~cm}^{-1}$, while for the surrounding pixels (labeled by yellow stars), only features originated from the $2 \mathrm{PySH}$ and/or $4 \mathrm{PySH}$ can be observed. More locations showing similar behavior are displayed in Figure S6. Therefore, the spatial resolution of the current system could be established to ca. 2 nm, which in this case corresponds to single-molecule spatial resolution, since the diameter of the CoTPP molecule is also $c a .2 \mathrm{~nm} .{ }^{15}$ 

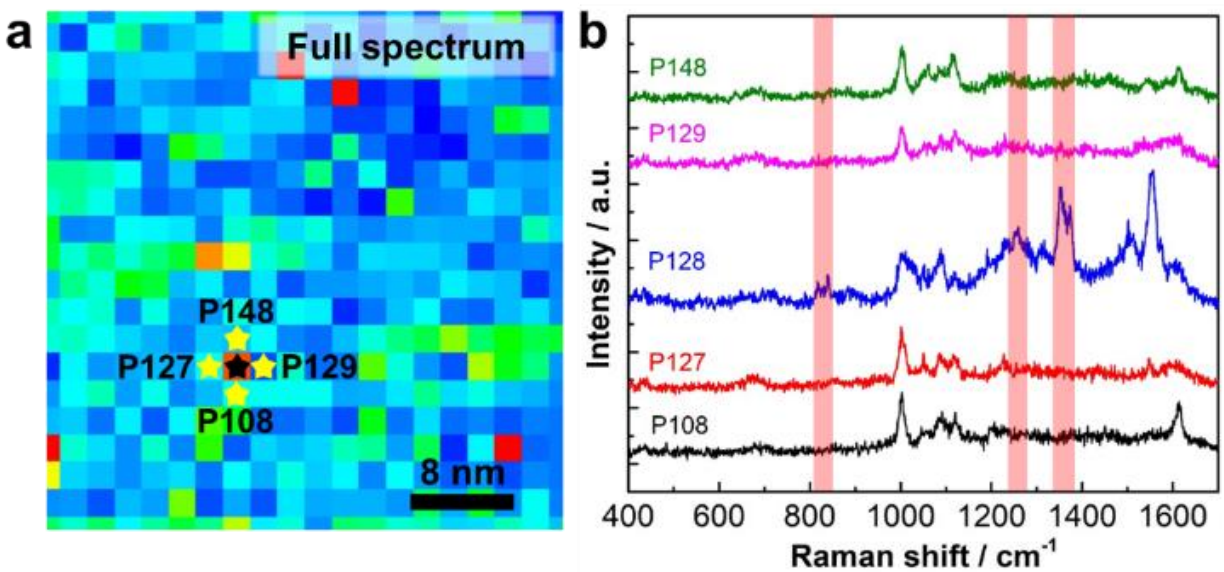

Figure 3. (a) TERS intensity map of the full spectrum of CoTPP on a (2PySH-4PySH)-modified TS-Au surface. The size of the map is $40 \times 40 \mathrm{~nm}^{2}$ with a pixel size of $2 \mathrm{~nm}$. (b) Corresponding TER spectra at the positions outlined in a. The exposure time per spectrum was $1 \mathrm{~s}$.

In order to further analyze the molecular orientation of the CoTPP-4PySH coordination complex, high-resolution TER mapping was performed on an atomically flat $\mathrm{Au}(111)$ surface. Figure 4a shows a TERS map with a pixel size of $2.5 \mathrm{~nm}$. Three typical TER spectra of the CoTPP-4PySH complex were extracted from the map. For type A, as highlighted by the red stripes, the intensity of the band at $\sim 835 \mathrm{~cm}^{-1}$ is higher than that of bands at $\sim 1000-1050 \mathrm{~cm}^{-1}$ and $\sim 1350-1370 \mathrm{~cm}^{-1}$. The spectra of type B is different from those of type A: the band at $\sim 835$ $\mathrm{cm}^{-1}$ is less intense than that of $\sim 1000-1050 \mathrm{~cm}^{-1}$ and $\sim 1350-1370 \mathrm{~cm}^{-1}$. Furthermore, for type $\mathrm{C}$, the peaks at $\sim 835 \mathrm{~cm}^{-1}$ and $\sim 1000-1050$ $\mathrm{cm}^{-1}$ become very weak while the band at $\sim 1350-1370 \mathrm{~cm}^{-1}$ becomes the dominant peak.
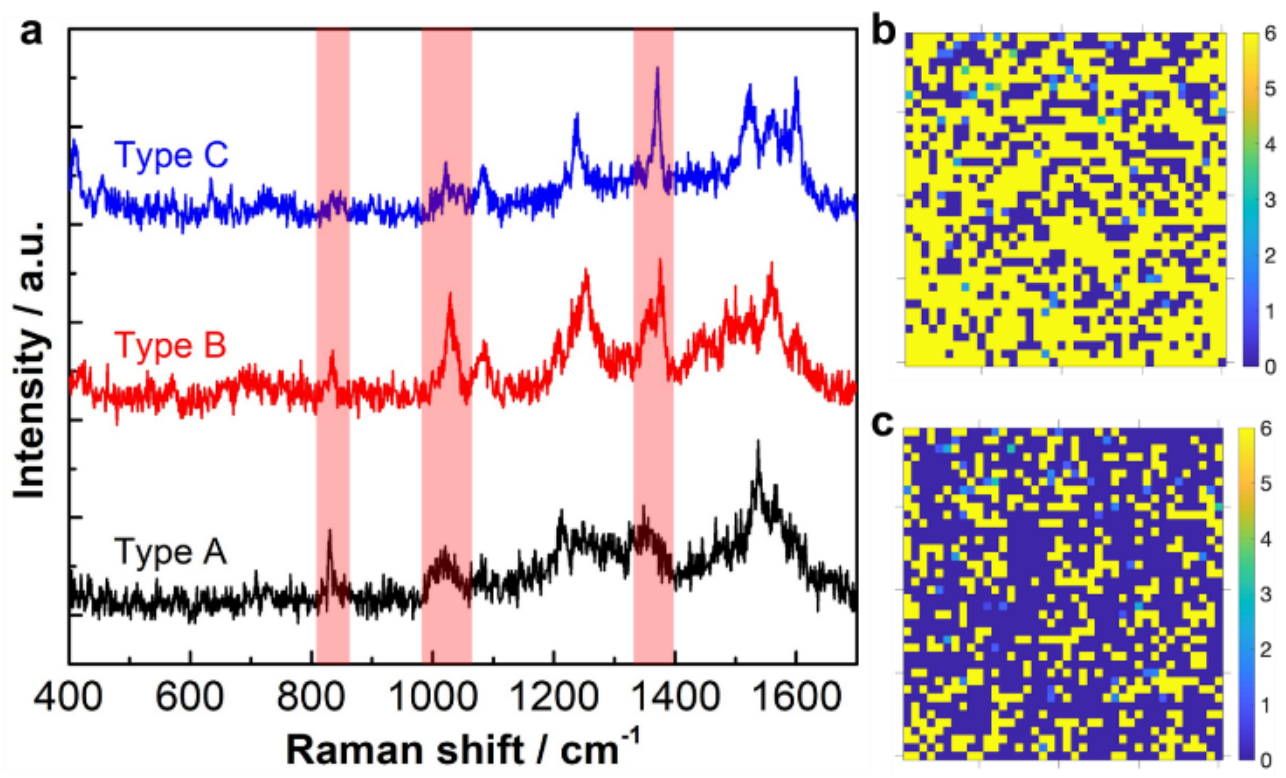

Figure 4. (a) Three typical TER spectra showing distinctive peak intensities of the bands highlighted by red stripes. (b) TERS intensity ratio (1370 $\mathrm{cm}^{-1}$ peak $/ 835 \mathrm{~cm}^{-1}$ peak) map of a CoTPP-4PySH complex on Au(111) surface. (c) TERS intensity ratio $\left(1020 \mathrm{~cm}^{-1}\right.$ peak $/ 835 \mathrm{~cm}^{-1}$ peak) map of a CoTPP-4PySH complex on $\mathrm{Au}(111)$ surface. The size of the map is $100 \times 100 \mathrm{~nm}^{2}$ with a pixel size of $2.5 \mathrm{~nm}$.

Figure 4b,c displays the TERS intensity ratio maps $\left(1370 \mathrm{~cm}^{-1}\right.$ peak $/ 835 \mathrm{~cm}^{-1}$ peak $)$ and $\left(1020 \mathrm{~cm}^{-1}\right.$ peak $/ 835 \mathrm{~cm}^{-1}$ peak) of the coordination species on $\mathrm{Au}(111)$, which reflects the relative distribution of the spectra of type $\mathrm{C}$ and type $\mathrm{B}$, respectively. It is found that $c a$. $64 \%$ of CoTPP-4PySH species on the $\mathrm{Au}(111)$ surface generate spectra of type $\mathrm{C}$ while $\mathrm{ca}$. $30 \%$ of the coordination complexes show spectra of type B. Only few pixels with type A can be extracted from the map (Figure S7b). More TER spectra of these three types at different locations are supplied in Figure S7. The observed spectral differences regarding the peak intensity of these bands among these four types of spectra suggest that the corresponding vibrational modes of these bands are enhanced to different degrees from type A to type C.

In STM-TERS, the local enhanced electromagnetic field is considered to be perpendicular to the conductive substrate and primarily boosts the out-of-plane Raman modes, which is well-established as the TER surface selection rule. In addition, according to theoretical simulations, when a $4 \mathrm{PySH}$ molecule adsorbs on a $\mathrm{Au}(111)$ surface, it can orient with different tilt angles on different sites. ${ }^{16}$ Thus, we propose that such 
spectral differences reflect an orientation difference of the CoTPP-4PySH coordination species. As the 4PySH adlayer was prepared by dropcasting method, the molecular orientation of $4 \mathrm{PySH}$ varied at different places, and therefore the orientation of CoTPP on top of them is also affected.

In order to verify our hypothesis, we performed DFT calculations to obtain the simulated Raman spectra of the CoTPP-4PySH coordination complex with different tilt angles. Figure 5a-d displays the schematic and the calculated Raman spectra of a CoTPP-4PySH complex with different tilt angles. We can see that the vibrational fingerprints of the CoTPP-4PySH coordination species are dependent on the molecular orientation. As outlined by the red stripes, the intensity of the band at $\sim 820-840 \mathrm{~cm}^{-1}$ will decrease when the tilt angle of CoTPP relative to the substrate is gradually rotated from $90^{\circ}$ to $0^{\circ}$. It is the dominant peak when the tilt angle is $90^{\circ}$. However, it becomes almost invisible if the tilt angle is reduced to $45^{\circ}$. This is because this peak is mainly related to the out-of-plane vibrational mode of the porphyrin core and phenyl rings, which will be weakened with decreasing tilt angles. The bands at $\sim 1000-1050 \mathrm{~cm}^{-1}$ and $\sim 1350-1370 \mathrm{~cm}^{-1}$ are associated with combination modes of out-plane and in-plane vibrations of the porphyrin core and phenyl rings. The intensities of these two bands increase gradually when the CoTPP-4PySH is rotated from $90^{\circ}$ to $60^{\circ}$, and then decrease after the tilt angle is decrease further from $60^{\circ}$ to $0^{\circ}$. The simulated Raman spectra of the CoTPP-4PySH with small steps of tilt angle from $30^{\circ}$ to $90^{\circ}$ are also displayed in Figure S8. By combining the experimental and simulation results, we are able to assign the observed spectra to a certain tilt angle range. For instance, the tilt angles of CoTPP with a Raman fingerprint from type A to type B to type C are corresponded to tilt angle at $c a .90^{\circ}, 60^{\circ}$, and $45^{\circ}$, respectively.

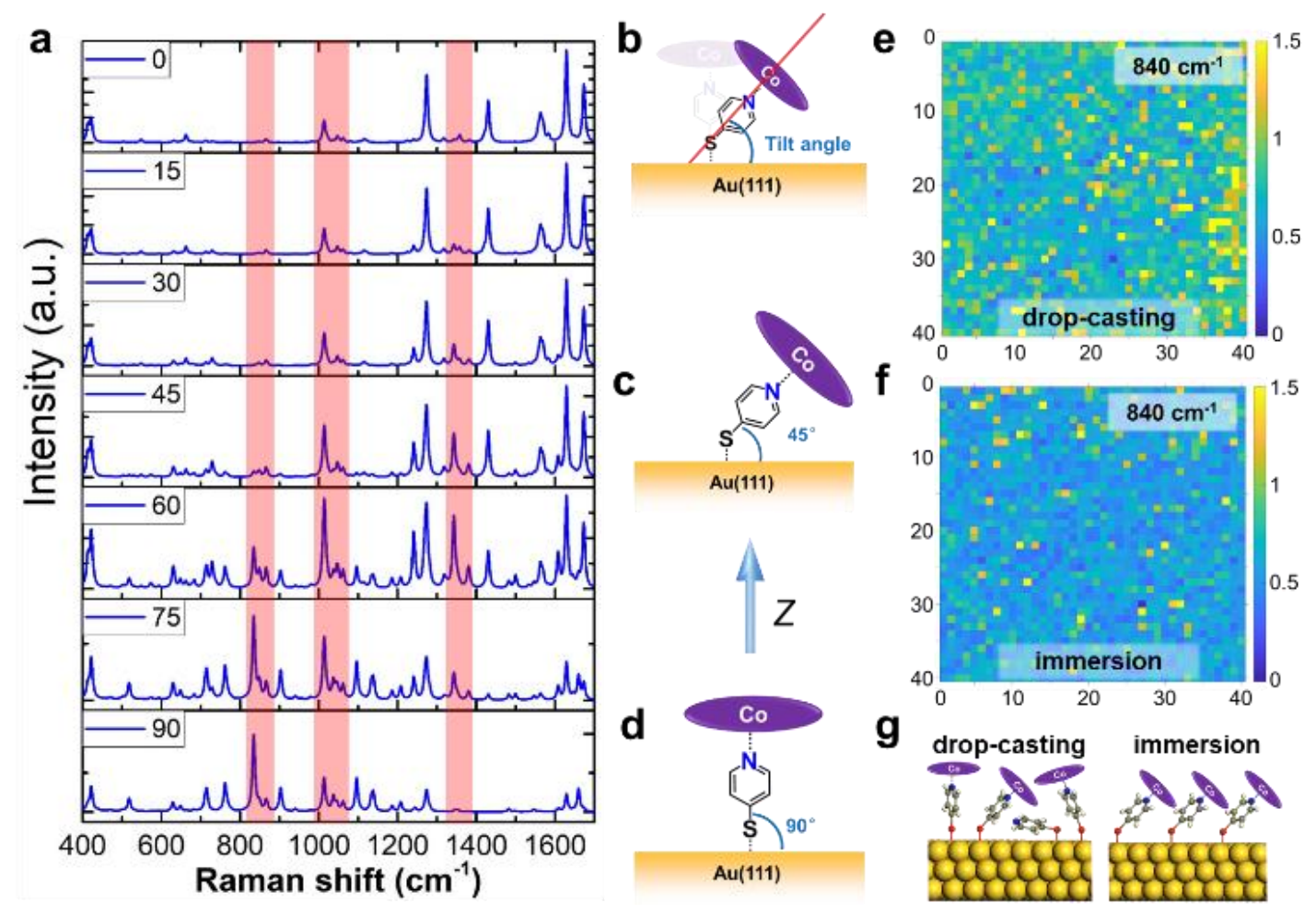

Figure 5. (a) Calculated Raman spectra of a CoTPP-4PySH-Au 2 complex with changes in the tilt angles showing dramatic spectral variations. (b) Schematic of the referring tilt angle of a CoTPP-4PySH complex on Au(111). (c, d) Schematic of a CoTPP-4PySH complex with tilt angles of $90^{\circ}$ and $45^{\circ}$ on Au(111), respectively. (e, f) TERS peak intensity maps $\left(840 \mathrm{~cm}^{-1}\right)$ of CoTPP-4PySH modified $\mathrm{Au}(111)$ surfaces prepared via a drop-casting/immersion protocol. (g) Proposed molecular models show the different adsorption orientation of the bottom 4PySH adlayer.

On the basis of the spectral intensity relationship of coordination species with different tilt angles, control experiments were carried out to investigate the tilt angle by changing the sample preparation protocol. It has been well-established that the adsorption of organic thiolate molecules from solution is a time-dependent self-assembly process. ${ }^{17}$ The self-assembled structure of a thiolate monolayer will convert from relatively disordered to well-ordered domains with certain tilting angles relative to the plane of the surface when the adsorption time is gradually increased. Two samples were prepared via drop-casting and immersion methods. The reaction time for these samples was $c a$. 45 seconds and 5 minutes, respectively. Then CoTPP solution was added to the samples, followed by rinsing with ethanol. Figure 5e,f displays the corresponding TERS peak intensity maps at $c a .840 \mathrm{~cm}^{-1}$ of these two samples. More high-contrast pixels can be detected and irregularly distributed on the drop-cast sample surface, which indicates that more coordination species with relatively larger tilt angle were obtained. In contrast, for the immersion sample smaller tilt angle coordination species were favored and the orientations of the coordination species are more homogeneous. Structural models showing the different orientations of the CoTPP-4PySH coordination species were given in Figure $5 \mathrm{~g}$. Such results further support the spectroscopic visualization of the orientation of coordination species on the Au(111) surface. 


\section{CONCLUSIONS}

In summary, we have shown the CoTPP-4PySH coordination complex on the Au surfaces can be distinguished and well-resolved on the molecular scale using STM-TERS. The spatial resolution is established to be $c a .2 \mathrm{~nm}$ in the current model system. Besides, the adsorption configurations and orientations of the coordination complexes can also be determined by comparing their TER spectra with DFT simulated results. The ability to probe and distinguish metal-organic coordination species on catalyst surfaces at the sub-molecular level using spectroscopic fingerprints have promising application in the characterization of biological, chemical and dynamic processes/conversions on catalyst surfaces. Since STM-TERS can only measure conductive samples, atomic force microscopy (AFM)-TERS is an important complimentary tool for probing non-conductive coordination systems. We anticipate that in-situ molecular scale TERS imaging of coordination systems, like catalytically active proteins, molecular electrocatalysts, supramolecular enzyme mimics and metal-organic frameworks, will enable further elucidation of catalytic mechanisms at the molecular scale.

\section{ASSOCIATED CONTENT}

Supporting Information. $\& \& \& \&$ $\& \& \&$

\section{AUTHOR INFORMATION}

\section{Corresponding Author}

* zenobi@org.chem.ethz.ch; zhy2008@ustc.edu.cn

\section{Author Contributions}

\$These authors contributed equally.

\section{Notes}

The authors declare no competing financial interest.

The original data used in this publication are made available in a curated data archive at ETH Zurich (https://www.researchcollection. ethz.ch) under the DOI: 10.3929/ethz-b-000489723

\section{ACKNOWLEDGMENT}

We thank Dr. Naresh Kumar (ETH Zurich) for insightful discussions. We thank the High Performance Computing Team at ETH Zurich for help with DFT calculations and the ERC program (Grant \# 741431-2DNanoSpec) for financial support.

\section{REFERENCES}

(1) (a) Allendorf, M. D.; Bauer, C. A.; Bhakta, R. K.; Houk, R. J. T. Luminescent metal-organic frameworks. Chem. Soc. Rev. 2009, 38, 1330. (b) Kalyanasundaram, K.; Grätzel, M. Applications of functionalized transition metal complexes in photonic and optoelectronic devices. Coord. Chem. Rev. 1998, 177, 347. (c) Evans, R. C.; Douglas, P.; Winscom, C. J. Coordination complexes exhibiting roomtemperature phosphorescence: Evaluation of their suitability as triplet emitters in organic light emitting diodes. Coord. Chem. Rev. 2006, 250, 2093.

(2) (a) Merlau, M. L.; del Pilar Mejia, M.; Nguyen, S. T.; Hupp, J. T. Artificial Enzymes Formed through Directed Assembly of Molecular Square Encapsulated Epoxidation Catalysts. Angew. Chem. Int. Ed. 2001, 40, 4239. (b) Jahan, M.; Bao, Q.; Loh, K. P. Electrocatalytically Active Graphene-Porphyrin MOF Composite for Oxygen Reduction Reaction. J. Am. Chem. Soc. 2012, 134, 6707. (c) Lin, S.; Diercks, C. S.; Zhang, Y. B.; Kornienko, N.; Nichols, E. M.; Zhao, Y. B.; Paris, A. R.; Kim, D.; Yang, P.; Yaghi, O. M.; Chang, C. J. Covalent organic frameworks comprising cobalt porphyrins for catalytic CO2 reduction in water. Science 2015, 349, 1208. (d) Feiters, M. C.; Rowan, A. E.; Nolte, R. J. M. From simple to supramolecular cytochrome P450 mimics. Chem. Soc. Rev. 2000, 29, 375. (e) Meunier, B. Metalloporphyrins as versatile catalysts for oxidation reactions and oxidative DNA cleavage. Chem. Rev. 1992, 92, 1411. (f) Morris, A. J.; Meyer, G. J.; Fujita, E. Molecular Approaches to the Photocatalytic Reduction of Carbon Dioxide for Solar Fuels. Acc. Chem. Res. 2009, $42,1983$.

(3) (a) Offord, D. A.; Sachs, S. B.; Ennis, M. S.; Eberspacher, T. A.; Griffin, J. H.; Chidsey, C. E. D.; Collman, J. P. Synthesi s and Properties of Metalloporphyrin Monolayers and Stacked Multilayers Bound to an Electrode via Site Specific Axial Ligation to a SelfAssembled Monolayer. J. Am. Chem. Soc. 1998, 120, 4478. (b) Kim, M.; Dygas, M.; Sobolev, Y. I.; Beker, W.; Zhuang, Q.; Klucznik, T.; Ahumada, G.; Ahumada, J. C.; Grzybowski, B. A. On-Nanoparticle Gating Units Render an Ordinary Catalyst Substrate- and Site-Selective. J. Am. Chem. Soc. 2021, 143, 1807.

(4) (a) Eckermann, A. L.; Feld, D. J.; Shaw, J. A.; Meade, T. J. Electrochemistry of redox-active self-assembled monolayers. Coord. Chem. Rev. 2010, 254, 1769. (b) Ponce, I.; Silva, J. F.; Onate, R.; Rezende, M. C.; Paez, M. A.; Zagal, J. H.; Pavez, J.; Mendizabal, F.; Miranda-Rojas, S.; Munoz-Castro, A.; Arratia-Perez, R. Enhancement of the Catalytic Activity of Fe Phthalocyanine for the Reduction of 
O-2 Anchored to Au(111) via Conjugated Self-Assembled Mono layers of Aromatic Thiols As Compared to Cu Phthalocyanine. J. Phys. Chem. C 2012, 116, 15329.

(5) (a) den Boer, D.; Li, M.; Habets, T.; Iavicoli, P.; Rowan, A. E.; Nolte, R. J. M.; Speller, S.; Amabilino, D. B.; De Feyter, S.; Elemans, J. Detection of different oxidation states of individual manganese porphyrins during their reaction with oxygen at a solid/liquid interface. Nat. Chem. 2013, 5, 621. (b) De Feyter, S.; De Schryver, F. C. Two-dimensional supramolecular self-assembly probed by scanning tunneling microscopy. Chem. Soc. Rev. 2003, 32, 139. (c) Wan, L. J. Fabricating and controlling molecular self-organization at solid surfaces: Studies by scanning tunneling microscopy. Acc. Chem. Res. 2006, 39, 334.

(6) (a) Kobayashi, Y.; Sato, S.; Uosaki, K.; Motobayashi, K.; Ikeda, K. Atomistic Control of Metal-Molecule Junctions for Efficient Photo-Induced Uphill Charge Transfer. J. Phys. Chem. C 2020, 124, 18173. (b) Collman, J. P.; Ghosh, S.; Dey, A.; Decréau, R. A.; Yang, Y. Catalytic Reduction of $\mathrm{O} 2$ by Cytochrome c Using a Synthetic Model of Cytochrome c Oxidase. J. Am. Chem. Soc. 2009, 131 , 5034.

(7) (a) Knecht, P.; Zhang, B.; Reichert, J.; Duncan, D. A.; Schwarz, M.; Haag, F.; Ryan, P. T. P.; Lee, T.-L.; Deimel, P. S.; Feulner, P.; Allegretti, F.; Auwärter, W.; Médard, G.; Seitsonen, A. P.; Barth, J. V.; Papageorgiou, A. C. Assembly and Manipulation of a Prototypical N-Heterocyclic Carbene with a Metalloporphyrin Pedestal on a Solid Surface. J. Am. Chem. Soc. 2021, 143, 4433. (b) Nandi, G.; Chilukuri, B.; Hipps, K. W.; Mazur, U. Surface directed reversible imidazole ligation to nickel(II) octaethylporphyrin at the solution/solid interface: a single molecule level study. Phys. Chem. Chem. Phys. 2016, 18, 20819. (c) Visser, J.; Katsonis, N.; Vicario, J.; Feringa, B. L. TwoDimensional Molecular Patterning by Surface-Enhanced Zn-Porphyrin Coordination. Langmuir 2009, 25, 5980. (d) Williams, F. J.; Vaughan, O. P. H.; Knox, K. J.; Bampos, N.; Lambert, R. M. First observation of capping/uncapping by a ligand of a Zn porphyrin adsorbed on $\operatorname{Ag}(100)$. Chem. Commun. 2004, 1688.

(8) Vaughan, O. P. H.; Williams, F. J.; Bampos, N.; Lambert, R. M. A chemically switchable molecular pinwheel. Angew. Chem., Int. Ed. 2006, 45, 3779 .

(9) (a) Stöckle, R. M.; Suh, Y. D.; Deckert, V.; Zenobi, R. Nanoscale chemical analysis by tip-enhanced Raman spectroscopy. Chem. Phys. Lett. 2000, 318, 131. (b) Pettinger, B.; Ren, B.; Picardi, G.; Schuster, R.; Ertl, G. Nanoscale probing of adsorbed species by tipenhanced Raman spectroscopy. Phys. Rev. Lett. 2004, 92, 4. (c) Stadler, J.; Schmid, T.; Zenobi, R. Nanoscale Chemical Imaging of SingleLayer Graphene. ACS Nano 2011, 5, 8442. (d) Kumar, N.; Wondergem, C. S.; Wain, A. J.; Weckhuysen, B. M. In Situ Nanoscale Investigation of Catalytic Reactions in the Liquid Phase Using Zirconia-Protected Tip-Enhanced Raman Spectroscopy Probes. J. Phys. Chem. L 2019, 10, 1669. (e) Kurouski, D.; Dazzi, A.; Zenobi, R.; Centrone, A. Infrared and Raman chemical imaging and spectroscopy at the nanoscale. Chem. Soc. Rev. 2020, 49, 3315. (f) Shao, F.; Zenobi, R. Tip-enhanced Raman spectroscopy: principles, practice, and applications to nanospectroscopic imaging of 2D materials. Anal. Bioanal. Chem. 2019, 411, 37. (g) Wang, X.; Huang, S.-C.; Huang, T.-X.; Su, H.-S.; Zhong, J.-H.; Zeng, Z.-C.; Li, M.-H.; Ren, B. Tip-enhanced Raman spectroscopy for surfaces and interfaces. Chem. Soc. Rev. 2017, 46, 4020 .

(10) (a) Chen, C.; Hayazawa, N.; Kawata, S. A $1.7 \mathrm{~nm}$ resolution chemical analysis of carbon nanotubes by tip-enhanced Raman imaging in the ambient. Nat. Commun. 2014, 5, 3312. (b) Su, H.-S.; Zhang, X.-G.; Sun, J.-J.; Jin, X.; Wu, D.-Y.; Lian, X.-B.; Zhong, J.-H.; Ren, B. Real-Space Observation of Atomic Site-Specific Electronic Properties of a Pt Nanoisland/Au(111) Bimetallic Surface by TipEnhanced Raman Spectroscopy. Angew. Chem. Int. Ed. 2018, 57, 13177. (c) Zhong, J.-H.; Jin, X.; Meng, L.; Wang, X.; Su, H.-S.; Yang, Z.L.; Williams, C. T.; Ren, B. Probing the electronic and catalytic properties of a bimetallic surface with $3 \mathrm{~nm}$ resolution. Nat. Nano. 2017, $12,132$.

(11) (a) Lee, J.; Crampton, K. T.; Tallarida, N.; Apkarian, V. A. Visualizing vibrational normal modes of a single molecule with atomically confined light. Nature 2019, 568, 78. (b) Lee, J.; Tallarida, N.; Chen, X.; Liu, P.; Jensen, L.; Apkarian, V. A. Tip-Enhanced Raman Spectromicroscopy of Co(II)-Tetraphenylporphyrin on Au(111): Toward the Chemists' Microscope. ACS Nano 2017, 11, 11466. (c) Jiang, S.; Zhang, Y.; Zhang, R.; Hu, C.; Liao, M.; Luo, Y.; Yang, J.; Dong, Z.; Hou, J. G. Distinguishing adjacent molecules on a surface using plasmon-enhanced Raman scattering. Nat. Nano. 2015, 10, 865. (d) Zhang, R.; Zhang, Y.; Dong, Z. C.; Jiang, S.; Zhang, C.; Chen, L. G.; Zhang, L.; Liao, Y.; Aizpurua, J.; Luo, Y.; Yang, J. L.; Hou, J. G. Chemical mapping of a single molecule by plasmon-enhanced Raman scattering. Nature 2013, 498, 82. (e) Jiang, N.; Foley, E. T.; Klingsporn, J. M.; Sonntag, M. D.; Valley, N. A.; Dieringer, J. A.; Seideman, T.; Schatz, G. C.; Hersam, M. C.; Van Duyne, R. P. Observation of Multiple Vibrational Modes in Ultrahigh Vacuum Tip-Enhanced Raman Spectroscopy Combined with Molecular-Resolution Scanning Tunneling Microscopy. Nano Lett. 2012, $12,5061$.

(12) (a) Jiang, S.; Chen, Z.; Chen, X.; Nguyen, D.; Mattei, M.; Goubert, G.; Van Duyne, R. P. Investigation of Cobalt Phthalocyanine at the Solid/Liquid Interface by Electrochemical Tip-Enhanced Raman Spectroscopy. J. Phys. Chem. C 2019, 123, 9852. (b) Chen, Z.; Jiang, S.; Kang, G.; Nguyen, D.; Schatz, G. C.; Van Duyne, R. P. Operando Characterization of Iron Phthalocyanine Deactivation during Oxygen Reduction Reaction Using Electrochemical Tip-Enhanced Raman Spectroscopy. J. Am. Chem. Soc. 2019.

(13) Domke, K. F.; Pettinger, B. In Situ Discrimination between Axially Complexed and Ligand-Free Co Porphyrin on Au(111) with Tip-Enhanced Raman Spectroscopy. ChemPhysChem 2009, 10, 1794.

(14) Li, C.-Y.; Duan, S.; Wen, B.-Y.; Li, S.-B.; Kathiresan, M.; Xie, L.-Q.; Chen, S.; Anema, J. R.; Mao, B.-W.; Luo, Y.; Tian, Z.-Q.; $\mathrm{Li}, \mathrm{J}$.-F. Observation of inhomogeneous plasmonic field distribution in a nanocavity. Nat. Nanotechnol. 2020.

(15) (a) Scudiero, L.; Barlow, D. E.; Mazur, U.; Hipps, K. W. Scanning Tunneling Microscopy, Orbital-Mediated Tunneling Spectroscopy, and Ultraviolet Photoelectron Spectroscopy of Metal(II) Tetraphenylporphyrins Deposited from Vapor. J. Am. Chem. Soc. 2001, 123, 4073. (b) Cai, Z.-F.; Wang, X.; Wang, D.; Wan, L.-J. Cobalt-Porphyrin-Catalyzed Oxygen Reduction Reaction: A Scanning Tunneling Microscopy Study. Chemelectrochem 2016, 3, 2048.

(16) Kučera, J.; Gross, A. Adsorption of 4-Mercaptopyridine on Au(111): A Periodic DFT Study. Langmuir 2008, $24,13985$.

(17) (a) Xu, S.; Cruchon-Dupeyrat, S. J. N.; Garno, J. C.; Liu, G.-Y.; Jennings, G. K.; Yong, T.-H.; Laibinis, P. E. In situ studies of thiol self-assembly on gold from solution using atomic force microscopy. J. Chem. Phys. 1998, 108, 5002. (b) Schreiber, F.; Eberhardt, A.; Leung, T. Y. B.; Schwartz, P.; Wetterer, S. M.; Lavrich, D. J.; Berman, L.; Fenter, P.; Eisenberger, P.; Scoles, G. Adsorption mechanisms, structures, and growth regimes of an archetypal self-assembling system: Decanethiol on Au(111). Phys. Rev. B 1998, $57,12476$. 
TOC:

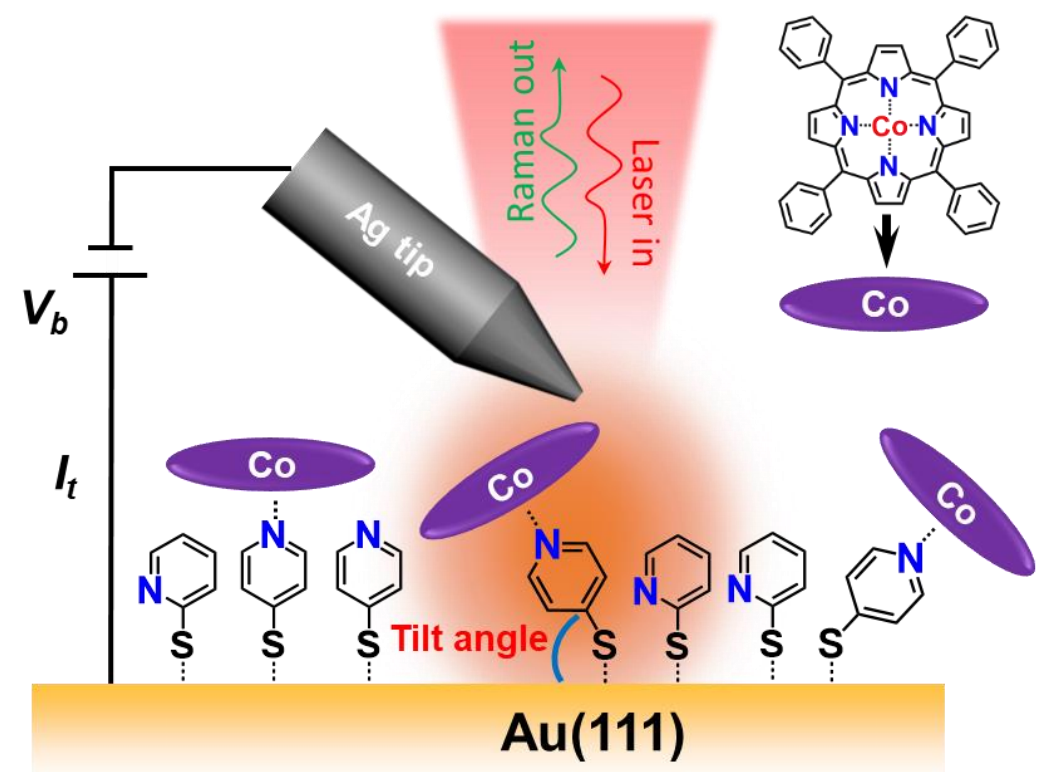

